\title{
Astrophotonics and IR astronomy
}

\author{
J. W. OByrne ${ }^{1}$, J. Bland-Hawthorn ${ }^{1}$, R. Haynes ${ }^{2}$, A. Horton ${ }^{2}$, \\ J. Bryant ${ }^{1}$ and J. G. Robertson ${ }^{1}$ \\ ${ }^{1}$ School of Physics, University of Sydney, ${ }^{2}$ Anglo-Australian Observatory
}

Working in collaboration with industry, the University of Sydney, the Anglo-Australian Observatory and Macquarie University are developing new 'astrophotonic' solutions to problems in astronomical instrumentation. A key first step involves overcoming the limitations imposed by multimode (MM) optical fibres that have been used by astronomers for many years to transport or reformat light from the telescope focus to an optical spectrograph. These large-core MM fibres maximise light into an astronomical instrument but at the expense of propagating many unpolarized modes. Until recently, this has deterred the use of more complex in-fibre processing of the light since this is typically limited to single-mode (SM) propagation. A MM to SM converter, known as a 'photonic lantern', was first demonstrated by Leon-Saval et al. (2005). If the number of transverse modes equals the number of SM fibres, and if a gradual and adiabatic transition between the MM fiber and the ensemble of SM fibres can be achieved, lossless coupling can take place in either propagation direction. Noordegraaf et al. (2009) demonstrated an efficient $1 \times 7$ photonic lantern (1 MM input and $7 \mathrm{SM}$ outputs) for the first time.

The application of a photonic lantern and a SM fibre device designed to suppress emission from hydroxyl molecules $(\mathrm{OH})$ in the Earth's upper atmosphere has recently been demonstrated in initial on-sky tests by Bland-Hawthorn et al. (2009). The OH-suppressing fibre, using a fibre Bragg grating, and a comparison fibre without a grating but also with a photonic lantern in series, were pointed at the sky. Light from the other ends of the fibres was re-imaged onto the focal plane of IRIS2 near-IR spectrograph/imager at the Anglo-Australian Telescope (AAT). The spectral region of interest was isolated with an $H$-band spectroscopic filter. The fibre Bragg gratings reduced the emission from $63 \mathrm{OH}$ lines to less than $1 \%$ at a resolving power of 10,000 although the suppressed region did not reach the dark sky limit because the fibres saw a 10 degree field of view and therefore includes the light from many stars. The experiment effectively cleaned up half of the spectroscopic $H$-band at low loss $(\sim 10 \%)$. A test of a complete $H$ and $J$-band $\mathrm{OH}$-suppressing fibre will be carried out later in the year.

This device can be applied in any project requiring spectra at near-IR wavelengths, for example high-redshift quasars or cool stars such as L and T dwarfs. A more detailed discussion of the scientific implications is presented by Ellis \& Bland-Hawthorn (2009).

Other photonics devices are also under development. In particular, an integrated photonic spectrograph (IPS) using an arrayed-waveguide grating (AWG) structure has recently had its first on-sky demonstration using IRIS2 in June 2009.

\section{References}

Bland-Hawthorn, J., Ellis, S. C., Haynes, R., \& Horton, A. 2009, AAO Newsletter No.115, 15 Ellis, S. C \& Bland-Hawthorn, J. 2008, MNRAS 386, 47

Leon-Saval, S. G., Birks, T. A., Bland-Hawthorn, J., \& Englund, M. A. 2005, Opt. Lett. 19, 2545

Noordegraaf, D., Skovgaard, P. M. W., Nielsen, M. D., \& Bland-Hawthorn, J. 2009, Opt. Express 17,1988 